Purdue University

Purdue e-Pubs

2009

\title{
Direct Simulation of Ionization and Ion Transport for Planar Microscale Ion Generation Devices
}

D. B. Go

T S. Fisher

S V. Garimella

Purdue Univ, sureshg@purdue.edu

Follow this and additional works at: https://docs.lib.purdue.edu/coolingpubs

Go, D. B.; Fisher, T S.; and Garimella, S V., "Direct Simulation of Ionization and Ion Transport for Planar Microscale Ion Generation Devices" (2009). CTRC Research Publications. Paper 114.

http://dx.doi.org/10.1088/0022-3727/42/5/055203

This document has been made available through Purdue e-Pubs, a service of the Purdue University Libraries. Please contact epubs@purdue.edu for additional information. 


\title{
Direct simulation of ionization and ion transport for planar
}

\section{microscale ion generation devices}

D B Go ${ }^{1}$, T S Fisher, and S V Garimella School of Mechanical Engineering and Birck Nanotechnology Center, Purdue University, West Lafayette, Indiana 47907 USA

\begin{abstract}
The theoretical performance of a planar microscale ion generation device is analyzed using the direct simulation Monte Carlo (DSMC) technique. The discrete motion and interactions of electrons and ions are modeled for atmospheric air as represented by $\mathrm{N}_{2}$ and $\mathrm{O}_{2}$. The ionization threshold of the device in air is found to be $70 \mathrm{~V}$ because of the effects of molecular excitations that reduce the energy of the free electrons and the nature of the collision cross-sections. Additionally, microscale planar ionization devices are revealed to be inherently inefficient because of the loss of electrons and ions to the dielectric boundary. A multiscale simulation of the electrohydrodynamics is also conducted by extracting the ion-neutral interactions from the DSMC calculations and integrating them into a continuum-scale fluid dynamics model. The multiscale simulations show that the ion-neutral body force distribution for the planar devices is concentrated on the face of the cathode and therefore limits the impact of the force on the flow. A scale analysis confirms that the body force distribution is insufficient to induce high flow rates at this scale.
\end{abstract}

${ }^{1}$ Corresponding author and currently Assistant Professor, Department of Aerospace and Mechanical Engineering, University of Notre Dame, Notre Dame, Indiana 46556, USA email: dgo@nd.edu 


\section{INTRODUCTION}

In recent years there has been increased interest in understanding the ionization and breakdown of atmospheric air between two electrodes spaced only a few to tens of microns apart [1-3]. For nearly a century, Paschen's law [4] has been used to establish breakdown criteria (i.e., the breakdown voltage for a given gap spacing and gas pressure); however these recent experiments have revealed that at the microscale the nature of the ionization fundamentally changes and deviates from Paschen's law. The authors' group [5] and others [6-10] have studied microscale ionization using Monte Carlo (MCC) collision particle simulations that incorporate the particle-in-cell (PIC) technique. Zhang et al. [5] were able to predict the deviation from Paschen's curve and reveal that electron field emission from the cathode is a significant electron source as the electrode spacing decreases to the microscale.

While the foregoing experiments [1-3] employed point-to-plane arrangements, the ionization between planar electrodes, where both the electrodes are on the surface of a substrate, has also been a topic that has received increased attention [11-14]. Because electronic devices and microelectromechanical systems (MEMS) are being scaled down, the distances between adjacent electrodes have decreased, with the possibility that air breakdown between these adjacent electrodes may be an unintended consequence of operating these devices at higher voltages. These recent studies [11-14] have investigated a number of electrode materials, including doped silicon ( $\mathrm{Si}$ ), polysilicon, and conventional metals such aluminum and silver, to understand the breakdown of air for planar geometries. In general, these studies focused on the unintended breakdown of air between planar electrodes.

However, other efforts have considered the design of planar ion generation devices for applications such as gas sensing and flow generation. The authors have previously investigated highly graphitic polycrystalline diamond (HGPD) as an electrode material for devices that use microscale gaps but operate below the breakdown threshold [15] and have developed planar microscale HGPD ion generation devices and compared them to titanium (Ti) devices [16]. While the Ti devices uncontrollably transitioned to breakdown, resulting in spark discharges, the HGPD devices operated at $100 \mathrm{nA}$ to $5 \mu \mathrm{A}$ currents and never transitioned to the breakdown regime. Chua et al. [17] designed a planar ionization device that used a millimeter-scale gap and macroscale, corona-discharge ionization as a possible gas sensor. They designed and microfabricated pin and collecting grid devices in which the electrode gaps ranged from 1.4 to $2.2 \mathrm{~mm}$. 
The devices were operated in negative corona discharge mode and a current of nearly $50 \mu \mathrm{A}$ was obtained prior to breakdown.

In addition to the proposed gas sensing application of Chua et al. [17], macroscale planar devices, called plasma actuators, have been extensively studied for aerodynamic flow control applications such as drag and acoustic signal reduction. Both dc corona and glow discharges [18-21] and ac dielectric barrier discharges [22-26] have been experimentally demonstrated to generate a flow or modify an external, bulk flow. The authors have previously studied macroscale planar configurations for heat transfer enhancement in the presence of an external bulk flow and reported over a $200 \%$ increase in the local heat transfer coefficient using a dc corona discharge and electrode gaps of 3-5 mm [27,28]. In addition to experiments, the modeling of macroscale, dc corona discharges using continuum-scale equations has been studied [29-33]. Continuum-scale models were applied to understand the ion transport and electrohydrodynamics in the planar configuration, and the simulated heat transfer response matched the experiments well [27].

Computational simulations of microscale planar configurations that used continuum-scale equations have also suggested that electrohydrodynamics effects can be scaled down for applications including an air pump and a heat transfer enhancement device for electronics cooling [34,35].

The present work presents the numerical simulation of a planar, microscale ion generation device with an electrode gap of $10 \mu \mathrm{m}$. A direct simulation Monte Carlo (DSMC) technique is employed to model ionization and charge motion (electrons and ions) in atmospheric pressure air (represented by $\mathrm{N}_{2}$ and $\mathrm{O}_{2}$ ). Studies on the effects of the emission current as well as the applied potential are used to understand the operational feasibility of microscale ion generation devices that are designed to operate below the breakdown threshold. Finally, the DSMC model is coupled to a Navier-Stokes model to simulate the microscale electrohydrodynamics.

\section{DIRECT SIMULATION MONTE CARLO METHOD}

\subsection{Overview}

The direct simulation Monte Carlo (DSMC) method is a particle simulation technique that has been widely used to study ionization and plasma transport [36,37]. The DSMC method simulates many (hundreds 
of thousands to millions of) particles, and probabilistically predicts collision events and ionization of neutral particles. Particle-in-cell direct simulation Monte Carlo (PIC-DSMC) is a specific type of DSMC approach that utilizes a self-consistent PIC method [38] to evaluate the electric field when it is altered by the presence of charged particles [39]. The PIC-DSMC method has been employed by Font [40] to investigate the performance and active mechanisms in dielectric barrier discharge (DBD) plasma actuators. Font et al. [41] also incorporated the results of the PIC-DSMC model into a continuum-scale model of the Navier-Stokes equations in order to predict flow evolution resulting from the forces extracted from the PIC-DSMC simulation. Zhang et al. [5] used the PIC-DSMC method to analyze microscale ionization of air due to fieldemitted electrons.

The DSMC method employed here derives from the work of Zhang et al. [5] and follows the nullcollision method of Vahedi and Surrendra [42]; the reader is directed to these articles for more information on this technique. The motion of electrons, $\mathrm{N}_{2}$ ions, and $\mathrm{O}_{2}$ ions in an ambient air atmosphere is simulated. Electrons are introduced into the simulation domain by electron field emission from the cathode and move through the domain toward the anode incrementally in discrete time steps. The electron motion is based on integrating Newton's second law in which the accelerating force is the applied electric field. The electrons statistically collide with neutral molecules and probabilistically undergo one of three events: elastic collision, excitation of the neutral, and ionization of the neutral. If an ion is generated, another electron is introduced into the system, and its motion is tracked. The ion is also introduced into the system and moves incrementally toward the cathode. The ion can also undergo collisions with neutral molecules (elastic or charge exchange), and these events are determined probabilistically as well. In these simulations, neutrals consist of $\mathrm{N}_{2}$ and $\mathrm{O}_{2}$ molecules only and are not modeled explicitly because they have no electrically induced motion. Additionally, the density of neutrals is very large relative to that of electrons and ions, and therefore all charged particles are assumed to interact only with neutral molecules. The neutral density was calculated based on the ideal gas law at atmospheric pressure and temperature and the ratio of $\mathrm{N}_{2}$ to $\mathrm{O}_{2}$ (approximately 3.75/1) in atmospheric air. Other trace components of air, such as the rare gases, carbon dioxide, and humidity are not considered but may play a role in the ionization and ion transport dynamics. Naturally occurring free electrons are also not modeled. 
The solution domain for the DSMC model is shown in figure 1 and represents the basic geometry of the planar engine. The electrodes are modeled as $1 \mu \mathrm{m}$ thick and $50 \mu \mathrm{m}$ wide with a gap spacing of $10 \mu \mathrm{m}$ [16]. The electrodes are placed on a dielectric layer. The left, right, and top boundaries are far-field boundaries and do not represent a physical surface. The electric field in the domain is determined by solving the homogeneous version of Poisson's equation by the finite volume method [42]:

$$
\nabla \cdot \vec{E}=-\nabla^{2} \Phi
$$

The full version of the electrostatic equation is typically inhomogeneous because it contains a space charge term related to the electric permittivity and particle density. In particle simulations, the inhomogeneous equation can be solved with a particle-in-cell simulation in which discrete charge densities are determined in each cell of the discretized domain after each iteration of particle motion; therefore, the electric field is dynamically computed. However, this approach introduces an extra computational step and can be very expensive in a simulation with a two-dimensional field. The assumption that the homogeneous equation is applicable can be rationalized by the magnitude of current. For every ion generated, an equal and opposite electron is created based on the conservation of charge. Therefore, the overall charge in the domain depends solely on the emitted current, and in general, the space charge effect is small unless the current is very high (order of several $\mathrm{mA}$ or greater depending both on geometry and electrode material) [43]. Because experimental devices typically have a much smaller current (HGPD devices operate at less than $10 \mu \mathrm{A}$ [16]) the space charge effect on the electric field is assumed to be negligible. However, this rationalization is valid only for the overall space charge in the domain. Local charge densities vary because electrons travel much faster than ions. A comparison between the present homogeneous approach and the particle-in-cell method by Zhang et al. [5] revealed that the predicted ion generation rates were nearly identical, thus lending confidence to the DSMC method used here.

The far-field boundaries and dielectric surface are modeled with homogeneous Neumann boundary conditions such that the electric field normal to these boundaries is zero. The surface boundaries of the two electrodes are given fixed values of applied potential: a positive value on the anode and a zero value (grounded) on the cathode. Electrons and ions that reach the boundaries are deleted from the simulation. Though surface charging may occur on the dielectric boundaries, this effect is assumed to be small and is not modeled here. Mesh independence of the potential is established for the microscale domain such that the left 
and right boundaries extend $100 \mu \mathrm{m}$ preceding and beyond the electrodes, respectively, and the top boundary is $125 \mu \mathrm{m}$ from the dielectric surface.

Electron field emission can be described by the Fowler-Nordheim equation [44] which gives the relationship between the current density $\left(j\right.$ in $\left.\mathrm{A} / \mathrm{cm}^{2}\right)$ and the applied electric field $(E$ in $\mathrm{V} / \mathrm{cm})$ :

$$
j=\frac{A \beta^{2} E^{2}}{\phi t^{2}(y)} \exp \left\lfloor-\frac{B \phi^{3 / 2} v(y)}{\beta E}\right\rfloor
$$

where $A$ and $B$ are Fowler-Nordheim constants [45], and $y, v(y)$, and $t^{2}(y)$ are functions that have wellestablished approximations [46]. The parameters $\beta$, the local field enhancement factor due to microscale features on the cathode surface, and $\phi$, the work function of the surface, are properties of the cathode material. Because experiments suggest HGPD as a favorable material for planar microscale ion generation devices [16], a work function of $\phi=4.60 \mathrm{eV}$ is used based on the work function of graphite, which has previously been used to represent the effective work function of highly graphitic polycrystalline diamond films [47]. In this work, $\beta$ is chosen so that a specific emission current is obtained, and therefore the impact of the emission current can be accurately studied. Because of the two-dimensional nature of the domain, the electric field normal at the surface of the cathode varies along the surface of the cathode. Therefore the emission current at the cathode was calculated for discretized cells in order to account for the spatial distribution of electron emission from the cathode. The summation of the discretized currents is equal to the fixed emission currents described herein. The emission area for each calculation was based on the area of the cell face and the span of the cathode assumed to be $1 \mathrm{~mm}$. The electrons are emitted randomly to match the current density and are given kinetic energies equal to $k_{\mathrm{B}} T$ with random direction where $k_{\mathrm{B}}$ is the Boltzmann constant.

\subsection{Benchmarking of Electron Motion and Ionization}

Three types of electron-neutral collisions - elastic, excitation of the neutral to a higher energy state, and ionization of the neutral—are modeled here. While including every single possible electron-neutral collision would be computationally impractical due to their sheer number, Zhang et al. [5] identified and listed 27 different collision reactions for nitrogen (one elastic, 23 excitations, and 3 positive ionizations) and 17 for oxygen (one elastic, 13 excitations, 1 negative ionization, and 3 positive ionizations) that are also 
included here. The collisions are treated as binary, and their probabilities are based on experimentally determined energy-dependent cross sections $(\sigma)$ and energy thresholds $\left(\varepsilon_{t h}\right)$ [48-51]. Figure 2 shows the collision cross sections for the basic ionization collisions of $\mathrm{N}_{2}$ and $\mathrm{O}_{2}$. The threshold energies required for these collisions are 15.6 and $12.0 \mathrm{eV}$, respectively. However, as the figure indicates, the maximum cross sections, and therefore the greatest probabilities of ionization, occur between 75 and $100 \mathrm{eV}$.

The DSMC simulation was benchmarked by predicting Townsend's first ionization coefficient, $\alpha$, for a one-dimensional electric field between two infinite flat plates using the method of Zhang et al. [5]. The computed values are compared to experimental results from Sanders [52] and the PIC-MCC simulations of Zhang et al. [5]. Figure 3 shows values of $\alpha$ for a range of applied electric fields where both values are normalized by pressure. Close agreement between the computational and experimental results suggests that the DSMC simulation accurately captures ionization physics for air. Additionally, the similarity between the results of the DSMC method here and the PIC-DSMC method of Zhang et al. [5] lends additional confidence to the use of the homogeneous Poisson equation, though it is not conclusive justification of the assumption.

Additional benchmarking is provided by calculation of the electron mean free path between collisions. Using 10,000 seed electrons, the mean free path was determined for a fixed field of $10^{7} \mathrm{~V} / \mathrm{m}(100$ $\mathrm{V}$ at $10 \mu \mathrm{m}$ ) between two infinite flat plates, and also for a $100 \mathrm{~V}$ applied potential for the physical domain in figure 1. The simulated mean free path between the flat plates was $370 \mathrm{~nm}$, a value that is similar to reported values that are in the range of 400 to $500 \mathrm{~nm}[3]$.

\subsection{Benchmarking of Ion Motion}

While the threshold energies and cross sections for various electron-neutral collisions have been well-cataloged experimentally, there are far fewer results for ion-neutral collisions. A number of ion-neutral collisions are possible, but the two most probable are elastic and charge exchange [53]. Zhang et al. [5] assumed a constant collision cross section of $10^{-18} \mathrm{~m}^{2}$ for all ion-neutral collisions $\left(\mathrm{N}_{2}{ }^{+} / \mathrm{N}_{2}, \mathrm{~N}_{2}{ }^{+} / \mathrm{O}_{2}, \mathrm{O}_{2}{ }^{+} / \mathrm{N}_{2}\right.$, $\mathrm{O}_{2}{ }^{+} / \mathrm{O}_{2}$ ) based on figure 13 of Vahedi and Surendra [39]. However, Vahedi and Surendra's [39] data are for $\mathrm{O}_{2}{ }^{+} / \mathrm{O}_{2}$ charge exchange collisions only. A second source of information is the atomic data tables of Ellis $e t$ al. [54] that give the momentum transfer collision integral $\Omega^{(1,1)}\left(\mathrm{T}_{\text {eff }}\right)$ (essentially, the energy averaged cross section) as a function of the effective ion-gas temperature (i.e., incident ion energy). The atomic data tables 
provide momentum exchange cross sections for both $\mathrm{N}_{2}{ }^{+} / \mathrm{N}_{2}$ and $\mathrm{O}_{2}{ }^{+} / \mathrm{O}_{2}$ collisions but only over a range of $0.319 \mathrm{eV}$. A second set of $\mathrm{N}_{2}{ }^{+} / \mathrm{N}_{2}$ data are available on the JILA website (a joint research institute between the University of Colorado and the National Institute of Standards and Technology) compiled by Phelps [55]. While this data set covers a range of $10,000 \mathrm{eV}$, it is not consistent with the atomic data tables.

Because the published data are inconsistent and somewhat incomplete, a constant collision cross section was employed for ion-neutral collisions. Two approaches were used to determine an appropriate cross section that correctly models the physics of ion motion in air. The first approach determined a collision cross section that produced the expected mean free path of the ions. The mean free path of ions in air at atmospheric pressure is approximately $60 \mathrm{~nm}$ based on the kinetic theory of gases [56]. A simulation using 10,000 ions was conducted that extrapolated the free path distribution in a fixed field of $10^{7} \mathrm{~V} / \mathrm{m}(100 \mathrm{~V}$ at $10 \mu \mathrm{m}$ ) over a range of cross sections as shown in figure 4 . It is apparent that a cross section of $3 \times 10^{-19} \mathrm{~m}^{2}$ gives a mean free path that is more consistent with the expected value. Notably, the approximation used by Zhang et al. [5] of $10^{-18} \mathrm{~m}^{2}$ results in an ion mean free path of approximately $15 \mathrm{~nm}$, which is much smaller than the expected value.

The second, more appropriate approach determined the ion mobility in air as a function of the collision cross section. The mobility of ions $\left(b\right.$ in $\left.\mathrm{m}^{2} / \mathrm{V}-\mathrm{s}\right)$ is the ratio of the ions' drift velocity to the magnitude of the applied electric field.

$$
b=\frac{v_{\text {ion }}}{E}
$$

The mobility can be determined directly by calculating the net velocity of an ion over several free paths (or collisions) and dividing by the applied electric field. The mobility of ions in air can be found in numerous studies in the literature with values ranging from $1 \times 10^{-4}$ to $4 \times 10^{-4} \mathrm{~m}^{2} / \mathrm{V}$-s $[34,57-60]$. The disparity in values is attributable to the differences in humidity, air purity, and the composition of the air used in the different studies. Figure 5 shows the average ion mobility calculated from simulations of 10,000 particles as a function of the collision cross section, as well as comparisons to values reported in the literature. The mobility generally decreases with increased collision cross section because collisions are more frequent (for the same reason, the mean free path decreases in figure 4). For a cross section of $10^{-18} \mathrm{~m}^{2}$, the mobility is actually outside the range of values reported in the literature. In order to provide an accurate estimate that is 
consistent with the mobility and mean free path data, a constant collision cross section of $3 \times 10^{-19} \mathrm{~m}^{2}$ was used in these simulations. While the energy variation in collision probability is not taken into account, the overall ion transport behavior of the system is predicted well using this approach.

\section{RESULTS OF DSMC CALCULATIONS}

\subsection{Ion Generation}

The transient variation of the electron current to both the collecting anode and the trapping dielectric between electrodes is shown in figure 6 for a fixed emission current of $5 \mu \mathrm{A}$ and applied potentials of 100 and $400 \mathrm{~V}$. The collecting electron current at the anode reaches a steady state more quickly at higher applied potentials because the acceleration force is greater. Additionally, the magnitude of the anode current increases with applied potential because more electrons are created through ionization events. At larger applied potentials, the energy of the system (and kinetic energy of the electrons) is higher, and therefore, ionization is more probable. In each case, there is significant electron loss at the dielectric layer primarily due to random collisions that alter the trajectory of the electrons. However, at $100 \mathrm{~V}$ the electron loss of nearly $75 \%$ is a far greater percentage of the total (anode + dielectric) current than it is at $400 \mathrm{~V}$, for which it is only $36 \%$. By increasing the applied potential, not only do emitted electrons traverse the domain more quickly (thus decreasing dielectric trapping), but more electrons are generated by ionization events that are not close to the boundary. What is apparent in the figure is that a planar ionization device is inherently inefficient because of the substantial electron loss at the boundary.

In order to quantify ionization in the $2 \mathrm{D}$ domain, an ionization yield parameter $(\xi)$ is defined as the number of ions formed per each electron emitted or

$$
\xi=\frac{\text { ions formed }}{\text { electron emitted }}
$$

Note that this metric is not equivalent to the number of ions formed by each emitted electron because electrons liberated by prior ionization events are able to ionize additional neutral molecules. For each simulation, the number of ions generated per emitted electron was tracked after the ionization (i.e., anode current) reached a steady state. 
Figure 7 shows the ionization yield as a function of applied potential for six fixed emission currents and clearly reveals that the ionization yield is independent of current. In other words, the normalized magnitude of ion generation does not depend on intensity of emission current although the absolute magnitude of ion generation does depend on emission current because there are more charged particles in the domain. By defining an ionization threshold as a yield of $\xi=0.001$ (or $0.1 \%$ ion yield), ionization is negligible until nearly $70 \mathrm{~V}$ of applied potential - a result that is somewhat unexpected considering the threshold energy required for ionization $\left(15.6 \mathrm{eV}\right.$ for $\mathrm{N}_{2}$ and $12.0 \mathrm{eV}$ for $\left.\mathrm{O}_{2}\right)$ as shown in figure 2. The highest probability of ionization is in the range of $70-100 \mathrm{eV}$, but the cross sections do not preclude the generation of ions at lower energies. The reason that little ionization is observed for lower potentials is likely due to energy retardation by non-ionizing collisions. In a vacuum, an electron emitted under a $20 \mathrm{~V}$ potential will reach the anode with a kinetic energy of $20 \mathrm{eV}$ - obtaining sufficient energy for ionization during travel. However, in a gaseous environment, an electron undergoes a number of excitation collisions that reduce its kinetic energy and generally act as an electron energy sink. This hypothesis is confirmed when the simulation is conducted under the artificial condition that no energy is lost during excitation collisions. Figure 8 shows a comparison of ionization yield for conditions in which the atmospheric physics are accurately modeled with those that are artificial. When energy loss is neglected, the ionization threshold decreases to $30 \mathrm{~V}$, and the exponential ionization yield takes a much steeper profile because the energies of the electrons are greater throughout the entire domain.

Figure 9 shows ion generation concentration contours for $100 \mathrm{~V}$ and $400 \mathrm{~V}$ at an emission current of $5 \mu \mathrm{A}$. In both figures 9 (a) and 9(b), the local ion generation concentrations have been normalized to their respective maximum values. For the $100 \mathrm{~V}$ case, ion concentration is negligible in a region within $2 \mu \mathrm{m}$ of the emitting cathode. However, under $400 \mathrm{~V}$ bias the first ionization events occur $0.5 \mu \mathrm{m}$ from the cathode because the accelerated electrons reach the ionization threshold energy in a much shorter distance. These distances match well with a scaling analysis for the distance required for the electrons to reach the threshold energy based on a 1D electric field approximation. Another significant difference is that under $100 \mathrm{~V}$ only one spatial region of peak ion generation is evident, but the contours for $400 \mathrm{~V}$ bias show two regions of peak ion generation. Peak ion generation occurs when the electron energy is near $70-100 \mathrm{eV}$ according to the peak cross sections. Electrons that experience ionization events lose a significant amount of kinetic 
energy, and under $100 \mathrm{~V}$ there is not sufficient distance for the ions to reaccelerate to energies that favor ionization given that they regularly lose some energy in excitation reactions. However, for an applied potential of $400 \mathrm{~V}$ there is sufficient space and therefore two peaks - one where originally emitted electrons are able to reach energies that favor ionization before other collisions occur, and a second where the reaccelerated and ejected electrons also reach such energies. Ultimately, at $400 \mathrm{~V}$ a significant percentage of the ions are generated farther from the cathode, and therefore these ions have more opportunities to exchange momentum with the neutral molecules.

\subsection{Ion Motion}

Figure 10 shows a comparison of the ion currents to the cathode and dielectric as opposed to the electron currents to the anode and dielectric for a fixed emission of $5 \mu \mathrm{A}$ and $400 \mathrm{~V}$. While the electron current to the anode stabilizes in $0.25 \mathrm{~ns}$, the ion current to the cathode requires much more time, nearly 25 ns, because the massive ions travel much more slowly. Additionally, the ion loss at the dielectric surface is much less significant than the electron loss ( $8 \%$ as compared to 35\%) because the ion cloud extends nearly 5 $\mu \mathrm{m}$ from the surface. Ions are not generally generated near the dielectric surface because near-surface electrons are lost to the boundary. It should be noted, however, that these percentages assume charge loss at the dielectric wall, and both surface charging and charge screening could affect these values. However, incorporation of such an effect would require a dynamic boundary condition that was considered too complex to incorporate at this time. Finally, the total electron current leaving the domain (to the anode and dielectric) is greater than the total ion current because there is a constant electron injection current due to emission and because not all electrons generate ions (particularly those lost to the dielectric).

The steady, normalized ion concentration, defined as $\rho_{\mathrm{ion}} / \rho_{\mathrm{in}, \max }$ where $\rho_{\mathrm{ion}}$ is in $\left[\mathrm{C} / \mathrm{m}^{3}\right]$, is shown in figure 11 for an applied potential of $400 \mathrm{~V}$. While the ion generation distribution has two peak regions, the motion of the ions results in a steady ion distribution that is highly concentrated near the edge of the cathode. Additionally, while most of the ion generation occurs within $7 \mu \mathrm{m}$ of the plate, the steady ion distribution extends nearly $10 \mu \mathrm{m}$ from the plate because of ion motion. In the same way that collisions alter the trajectory of ions such that they are lost to the dielectric, some ions (though proportionally less) also move away from the electrodes. As mentioned earlier, in macroscale simulations of positive corona discharges, an 
ion concentration is specified as a boundary condition at the anode. The ion density distribution throughout the domain is then determined by continuum-scale ion transport equations. For this reason, the ion density distribution of a corona discharge tends to be more uniform throughout the domain as the ions generated at one boundary (anode) all move towards the opposite boundary (cathode). In contrast, at the microscale, the ion generation occurs with discrete peaks at locations within the domain, rather than at a boundary, resulting in a less-uniform ion density distribution.

\section{ELECTROHYDRODYNAMICS}

\subsection{Multiscale Method}

A multiscale method is implemented here to study the efficacy of using dc ion generation at the microscale to generate a macroscale flow or ionic wind. The multiscale method uses the DSMC simulation to predict ion-neutral interactions and then translates these results into a continuum-scale, finite volume simulation of the electrohydrodynamics and heat transfer using the commercial software Fluent [61]. The multiscale method is similar to that implemented by Font et al. [41] to study macroscale ac dielectric barrier discharge plasma actuators.

An ionic wind is a flow generated from the motion of ions in a neutral atmosphere and is caused by the momentum exchange between ions and neutral molecules. The ion motion imposes a body force on the bulk flow which can be defined classically by a Coulombic force:

$$
\vec{f}=\rho_{\text {ion }} \vec{E}
$$

where $\rho_{\text {ion }}$ is the ion concentration. For this simulation, the force imparted on the neutral molecules is calculated directly from the discrete DSMC collisions after the ion current reaches a steady-state value. Body force contours (in $\mathrm{N} / \mathrm{m}^{3}$ ) are extracted from the individual momentum exchange interactions. The number of collisions that occur in a given mesh cell are summed, and the time-averaged force is determined based on the duration of the simulation after a steady state is achieved. With ion momentum change defined by $\Delta(m v)_{\text {ion }}$, the body force in a single cell is defined as

$$
\vec{f}_{\text {cell }}=-\frac{1}{t_{\mathrm{ss}}} \sum_{i=1}^{N} \frac{(m \vec{v})_{\text {ion }}}{\Delta x \Delta y \Delta z}
$$


where $N$ is the number of collisions in the cell, $\Delta x$ and $\Delta y$ are the dimensions of the cell, $\Delta z$ is the ion motion in the spanwise direction which is roughly equal to the $1 \mathrm{~mm}$ span of the electrodes, and $t_{\mathrm{ss}}$ is the time of the simulation after a steady state is achieved (10 ns in this work). The negative sign implies Newton's third law where the force on the neutral molecules is equal and opposite to the force on the ion. Figure 12 shows contours of the magnitude of the body force for a $400 \mathrm{~V}$ applied potential and a $50 \mu \mathrm{A}$ emission current. The full-size image includes stream traces to visualize how the force field follows the electric field from the anode to the cathode. The inset shows a magnified view near the cathode including the force vectors.

A few important observations can be made from this plot. The magnitude of the body force is highest very close to the cathode, and this location corresponds to the larger ion density concentrated near the cathode in figure 11. The magnitude of the body force decreases sharply from the peak location so that it is many orders of magnitude smaller with distance away from the electrodes. A second observation is that both the $x$ and $y$ components of the body force are significant as highlighted by the stream traces, and this may have the hydrodynamic effect of both accelerating the flow and pushing it downward toward the surface. However, in the region in which the body force is greatest, the ion motion is predominantly in the $x$ direction, corresponding to the approximately $1 \mathrm{D}$ electric field in the lateral plane between electrodes. In general, the magnitude and spatial extent of the extracted body force compare well with an earlier, continuum-scale study [35]. However, because of the nature of the ion generation and the electric field, both the body force and the energy generation are highly concentrated near the leading edge of the cathode.

\subsection{Multiscale Results}

The continuum-scale momentum and energy equations can still be utilized at the microscale based on the Knudsen number $(\mathrm{Kn})$ which compares the mean free path of the collisions $(\lambda)$ to the relevant length scale of the problem $(L)$ :

$$
\mathrm{Kn}=\frac{\lambda}{L}
$$

The mean free path of the ions and neutral molecules is $\lambda \approx 0.1 \mu \mathrm{m}$, and the relevant length scale is the gap between the electrodes $(L \approx 10 \mu \mathrm{m})$, giving a value of $\mathrm{Kn} \approx 0.01$, which is sufficiently small to justify the continuum-scale approximations. The body force calculated from the collisions in the DSMC simulation are 
integrated as momentum sources (in $x$ and $y$ directions) directly into Fluent by a user-defined function (UDF).

The Fluent model is intended to assess the impact of the DSMC-calculated body force on quiescent flow. In practice, modeling quiescence over external flat plates can be difficult, and, therefore, the simulation instead used a fixed free-stream flow condition where the inlet flow enters the domain with a very small uniform speed of $10^{-4} \mathrm{~m} / \mathrm{s}$. The same domain used for the DSMC simulations is also used here except that it is extended vertically to $700 \mu \mathrm{m}$ (more than twice the expected boundary layer thickness) in order to include the far-field flow. A domain height-independence study (including domain heights greater than 700 $\mu \mathrm{m})$ was conducted to ensure that the domain height used did not affect the final solution.

Figure 13 shows the extracted boundary layer profiles with and without the DSMC body forces for three locations along the flat plate. While the imposed boundary layer-like bulk flow profile is distorted by the presence of the ionic wind, the results reveal that the flow acceleration is minimal (less than $10^{-5} \mathrm{~m} / \mathrm{s}$ ), and much less than even the $10^{-4} \mathrm{~m} / \mathrm{s}$ flow used as the free-stream condition. The very small induced flow appears to be a result of the body force being highly concentrated within a region that is less than $1 \mu \mathrm{m}$ from the leading edge of the cathode. Therefore, the body force may not sufficiently perturb the flow over and above the passive disruption of the flow due to the presence of the cathode itself.

In order to interpret the effect of the ionic wind on the flow, a basic scale analysis is conducted based on a similar analysis for natural convection [62]. For this scale analysis, the flow is approximately described by the boundary layer equations, and any effects of Joule heating on the local air density are assumed negligible. Figure 14 illustrates the geometry considered in the scale analysis. The region of interest is the region over which the body force acts, as denoted by the axial region, $x_{\mathrm{EHD}}$, and the electrohydrodynamic boundary layer thickness, $\delta_{\mathrm{EHD}}$. The governing equations in this region, using the boundary layer approximation and including the Coulombic body force, are:

$$
\begin{gathered}
\text { continuity: } \frac{\partial u}{\partial x}+\frac{\partial v}{\partial y}=0 \\
\text { momentum: } u \frac{\partial u}{\partial x}+v \frac{\partial u}{\partial y}=\eta \frac{\partial^{2} u}{\partial y^{2}}+\frac{\rho_{\text {ion }} E}{\rho}
\end{gathered}
$$

Considering the scales, mass conservation leads to the following relationship 


$$
\frac{u}{x_{\mathrm{EHD}}} \sim \frac{v}{\delta_{\mathrm{EHD}}} \rightarrow v \sim \frac{u \delta_{\mathrm{EHD}}}{x_{\mathrm{EHD}}}
$$

The momentum equation considers three different terms - inertia, friction, and the body force — which may balance each other. Again considering the region over which the body force acts, the terms in the momentum equation also scale similarly, and the mass conservation relationship can be substituted for $v$ into the inertia term. Therefore, both the inertial terms reduce to the same scale relationship. Dividing through by the body force therefore yields the following scale relationships for inertia, friction, and Coulombic body force.

$$
\begin{array}{ccc}
\frac{\rho u^{2}}{\rho_{\text {ion }} E x_{\mathrm{EHD}}} & \frac{\eta \rho u}{\rho_{\text {ion }} E \delta_{\mathrm{EHD}}^{2}} & 1 \\
\text { inertia } & \text { friction } & \text { body force }
\end{array}
$$

Two different balances are possible. If the Coulombic body force is balanced by inertia, then the velocity, $u$ reduces to

$$
u \sim\left(\frac{\rho_{\mathrm{ion}} E x_{\mathrm{EHD}}}{\rho}\right)^{1 / 2}
$$

In this case the velocity is related to the square root of the body force which is the expected circumstance when the body force is converted efficiently into kinetic energy. However, in order to initiate flow near a boundary, the Coulombic body force must overcome strong friction forces. In this regime, the velocity reduces to

$$
u \sim \frac{\rho_{\mathrm{ion}} E \delta_{\mathrm{EHD}}^{2}}{\eta \rho}
$$

The velocity is now linearly related to the Coulombic body force but is quadratically related to the region over which the body force acts. Using both of these scales for velocity, characteristic velocities can be estimated. The density of air is of the order of magnitude of $1\left(\rho \sim 1 \mathrm{~kg} / \mathrm{m}^{3}\right)$, and the viscosity is on the order of $10^{-5}\left(\eta \sim 10^{-5} \mathrm{~m}^{2} / \mathrm{s}\right)$. The body force has a peak magnitude on the order of $10^{7}\left(\rho_{\mathrm{ion}} E \sim 10^{7} \mathrm{~N} / \mathrm{m}^{3}\right)$, but it occurs over a region smaller than $1 \mu \mathrm{m}$ and decreases throughout the domain. The $x$ and $y$ dimensions over which the body force acts are of similar magnitude as shown in the DSMC results $\left(\delta_{\mathrm{EHD}} \sim x_{E H D}\right)$. 
Figure 15 shows the approximate magnitude of the induced velocities predicted respectively by Eqs. (10) and (11) as a function of the region over which the body force acts. For very small regions of influence of less than $1 \mu \mathrm{m}$, the friction balance results in very low velocity. This general trend is consistent with that observed in the multiscale analysis for which the body force is highly concentrated at the front edge of the cathode. Though the magnitude of velocity from the simulations is actually smaller than that predicted from the scale analysis, this is likely due to the $2 \mathrm{D}$ nature of the body force field which actually impinges on the front edge of the cathode as well as from the obstruction posed by the protruding cathode. Still, the scale analysis helps to demonstrate that friction is likely the dominant force that prevents higher ionic wind flow rates at the microscale.

\section{CONCLUSIONS}

A planar microscale ion generation device has been analyzed using the direct simulation Monte Carlo technique. The DSMC simulations highlight how ionization yield is a strong function of the applied potential but independent of the emission current. Additionally, the simulations reveal that ionization does not effectively initiate until nearly $70 \mathrm{~V}$ of applied potential, and that this lower physical limit is a result of both ionization cross sections and the role that excitation reactions play in reducing the electron energy. Ion generation contour maps demonstrate how the applied potential affects the areas where ions are generated, with high potentials resulting in more than one location of high ion generation. Further, the dielectric surface plays a significant role in the transport at the microscale because there is significant electron loss to the boundary, and notable, though proportionally less, ion loss as well. Increasing the applied potential helps to mitigate the particle loss by increasing the speed of transport.

A multiscale simulation has also been conducted to understand the microscale electrohydrodynamics of the ion generation device. A body force distribution has been extracted from the discrete ion-neutral collisions in the DSMC simulation. The multiscale results show that the body force is insufficient to generate an appreciable flow rate, and a scale analysis confirms that the small region over which the body force peaks near the cathode is likely to be insufficient to generate higher flow rates.

\section{ACKNOWLEDGEMENT}


The authors wish to acknowledge Intel Corporation for financial support. 


\section{REFERENCES}

[1] Torres J-M and Dhariwal R S 2000 Nanotechnology 10 102-7

[2] Lee R-T, Chung H-H, and Chiou Y-C 2001 IEE Proc. - Science, Measurement and Technology 148 pp 8-14

[3] Slade P G and Taylor E D 2002 IEEE Trans. Compon. Packag. Technol. 25 390-396

[4] Paschen F 1889 Ann. Phys. 27369

[5] Zhang W, Fisher T S, and Garimella S V 2004 J. Appl. Phys. 96 6066-72

[6] Radmilović-Radjenović M, Lee J K, Iza F, and Park G Y 2005 J. Phys. D: Appl. Phys 38 950-4

[7] Radmilović-Radjenović M and Radjenović B 2007 Plasma Sources Sci. Technol. 16 337-40

[8] Radmilović-Radjenović M and Radjenović B 2007 IEEE Trans. Plasma Sci. 35 1223-8

[9] Radmilović-Radjenović M and Radjenović B 2008 Plasma Sources Sci. Technol. 17024005

[10] Radmilović-Radjenović M and Radjenović B 2008 Europhysics Lett. 8325001

[11] Ono T, Sim D Y, and Esashi M 2000 J. Micromech. Microeng. 10 445-51

[12] Wallash A and Levit L 2003 Proc. SPIE vol. 4980 p 87-96

[13] Chen C-H, Yeh J A, and Wang, P-J 2006 J. Micromech. Microeng. 16 p 1366-73

[14] Strong F W, Skinner J L, and Tien N C 2008 J. Micromech. Microeng. 18075025

[15] Peterson M S, Zhang W, Fisher T S, and Garimella S V 2005 Plasma Sources Sci. Technol. 14 654-60

[16] Go D B, Fisher T S, Garimella S V, Bahadur V, 2008 Plasma Sources Sci. Technol.- in review

[17] Chua B, Wexler A S, Tien N C, Niemeier D A, and Holmen B A 2008 J. Microelectromech. Syst. 17 $115-123$

[18] Léger L, Moreau E, and Touchard G G 2002 IEEE Trans. Indust. Appl. 38 1478-85

[19] Artana G, D’Adamo J, Léger L, Moreau E, and Touchard G G 2002 AIAA J. 40 1773-79

[20] Moreau E, Léger L, and Touchard G G 2006 J. Electrostatics 64 215-25

[21] Moreau E 2007 J. Phys. D: Appl. Phys. 40 605-36

[22] Roth J R, Sherman D M, and Wilkinson S P 2000 AIAA J. 38 1166-72

[23] Enloe C, McLaughlin T E, VanDyken R D, Kachner K D, Jumper E J and Corke T C 2004 AIAA J. 42 $589-94$ 
[24] Enloe C L, McLaughlin T E, VanDyken R D, Kachner K D, Jumper E J, Corke T C, Post M and Haddad O 2004 AIAA J. 42 595-604

[25] Corke T C, Post M L, Orlov D M 2007 Prog. Aerospace. Sci. 43 193-217

[26] Moreau E, Sosa R, and Artana G 2008 J. Phys. D: Appl. Phys. 41115204

[27] Go D B, Garimella S V, Fisher T S, and Mongia R K 2007 J. Appl. Phys. 102053302

[28] Go D B, Maturana R A, Garimella S V, and Fisher T S, 2008 Int. J. Heat Mass Transf. (Preprint doi:10.1016/j.ijheatmasstransfer.2008.05.012)

[29] El-Khabiry S and Colver G M 1997 Phys. Fluids 9 587-99

[30] Colver G M and El-Khabiry S 1999 IEEE Trans. Indust. Appl. 35 387-94

[31] Atten P, Adamiak K, Khaddoura B, and Coulomb J-H 2004 J. Optoelectronics Adv. Materials 6 102328

[32] Adamiak K and Atten P 2004 J. Electrostatics 61 85-98

[33] Tulita C, Benard E, and Raghunathan S 2006 J. Electrostatics 64 611-20

[34] Schlitz D J, Garimella S V and Fisher T S 2004 ASME Heat Transfer/Fluids Engineering Summer Conference (Charlotte, NC) 56470

[35] Go D B, Garimella S V, and Fisher T S 2006 Tenth Intersociety Conference on Thermal and Thermomechanical Phenomena in Electrical Systems (ITherm'06) (San Diego, CA) p 46-53

[36] Surendra, M, Graves, D B, and Jellum G M 1990 Phys. Rev. A 41 1112-25

[37] Boeuf J-P and Pitchford L C 1991 IEEE Trans. Plasma Sci. 19 286-96

[38] Birdsall C and Langdon A 1985 Plasma Physics via Computer Simulation (New York:McGraw-Hill)

[39] Vahedi V and Surendra M 1995 Computer Phys. Comm. 87 179-98

[40] Font G 2006 AIAA J. 44 1572-78

[41] Font G, Jung S, Enloe C L, McLaughlin T E, Morgan W L, and Baughn J W 2006 44th AIAA Aerospace Sciences Meeting and Exhibit (Reno, NV) AIAA 2006-167

[42] Patankar S V 1980 Numerical Heat Transfer and Fluid Flow (Washington D.C.: Hemisphere)

[43] Barbour J P, Dolan W W, Trolan J K, Martin E E, and Dyke W P 1953 Phys. Rev. 92 45-51

[44] Fowler R and Nordheim L 1928 Proc. R. Soc. London, Ser. A 119 626-39 
[45] Good R and Müller E 1956 Field Emission Encyclopedia of Physics vol 21, ed S. Flügge (Berlin:Springer) p 176-231

[46] Spindt C, Brodie I, Humphrey L, and Westerberg E 1976 J. Appl. Phys. 475248

[47] Gröning O, Nilsson L-O, Gröning P, and Schlapbach L 2001 Solid-State Electron. 45 929-44

[48] Lawton S A and Phelps A V 1978 J. Chem. Phys. 69 1055-68

[49] Straub H C, Renault P, Lindsay B G, Smith K A, and Stebbings R F 1996 Phys. Rev. A 54 2146-53

[50] Morgan W L, Boeuf J P, and Pitchford L C 1995-1998 The Siglo Database, CPAT and Kinema Software, http://www.siglo-kinema.com (accessed January 12, 2006)

[51] Morgan W L 2000 Adv. At. Mol. Opt. Phys. 43 79-110

[52] Sanders F H 1933 Phys Rev 44 1020-1024

[53] Phelps A V 1991 J. Phys. Chem. Ref. Data $20557-73$

[54] Ellis H W, Pai R Y, McDaniel E W, Mason E A, and Viehland L A 1976 Atomic Data Nuclear Data Tables 17 177-210

[55] Phelps A V 2002 Ion-Neutral Collision Cross-sections, http://jilawww.colorado.edu/ avp/collision_data/ionneutral/IONATOM.TXT (accessed June 12, 2008)

[56] Nasser E 1971 Fundamentals of Gaseous Ionization and Plasma Electronics (New York:WileyInterscience)

[57] Stuetzer O 1959 J. Appl. Phys. 30 984-94

[58] Robinson M 1961 Trans. Amer. Inst. Elec. Eng. (AIEE J.) 80 143-50

[59] Stearns R G 1990 J. Appl. Phys. 67 2789-99

[60] Owsenek B L, Seyed-Yagoobi J, and Page R H 1995 J. Heat Transf. 117 309-315

[61] Fluent Inc. 2003 Fluent 6.1 User's Guide (Lebanon, NH)

[62] Bejan A, 2004 Convection Heat Transfer, $3^{\text {rd }}$ Ed. (Hoboken, NJ:Wiley) 


\section{LIST OF FIGURES}

Figure 1 Schematic diagram of the basic simulation domain.

Figure 2 Collision cross sections as a function of electron energy for the ionization of $\mathrm{N}_{2}$ and $\mathrm{O}_{2}$. The inset shows a magnified view to clearly illustrate the minimum energy required for ionization.

Figure 3 DSMC values of Townsend's first ionization coefficient as compared to the experimental values of Sanders [52] and the PIC-MCC values of Zhang et al. [5].

Figure 4 Variation of mean free path with collision cross section for ion-neutral collisions.

Figure 5 Variation of ion mobility with collision cross section for ion-neutral collisions along with values reported from the literature [58,59].

Figure 6 Electron current evolutions with time for an emission current of $5 \mu \mathrm{A}$.

Figure 7 Ionization yield as a function of applied potential.

Figure 8 Ionization yield as a function of applied potential with the artificial condition of no energy loss during excitation reactions.

Figure 9 Ion generation concentration contours under applied potentials of (a) $100 \mathrm{~V}$, and (b) $400 \mathrm{~V}$, for an emission current of $5 \mu \mathrm{A}$. Each ion concentration plot is normalized to its respective maximum value. The insets are magnified views of the gap between the electrodes.

Figure 10 Ion and electron current evolution with time under a $400 \mathrm{~V}$ applied potential for an emission current of $5 \mu \mathrm{A}$.

Figure 11 Normalized, steady ion concentration contours, $\rho_{\text {ion }} / \rho_{\text {ion,max }}$, under a $400 \mathrm{~V}$ applied potential for an emission current of $5 \mu \mathrm{A}$. The inset is a magnified view of the gap between the electrodes.

Figure 12 Body force magnitude contours (in $\mathrm{N} / \mathrm{m}^{3}$ ) under a $400 \mathrm{~V}$ applied potential with a $50 \mu \mathrm{A}$ emission current. The inset is a magnified view of the gap between the electrodes.

Figure 13 Boundary layer profiles for quiescent air as approximated by $10^{-4} \mathrm{~m} / \mathrm{s}$ flow and a $400 \mathrm{~V} / 50 \mu \mathrm{A}$ emission current ionic wind at three locations. The locations are denoted by the small graphic on the plot.

Figure 14 Basic geometric definitions for the scale analysis.

Figure 15 Approximate magnitude of induced velocity as a function of the region of influence. The dashed line refers to the induced velocity when the friction forces are balanced by the Coulombic force, and the solid line refers to the induced velocity when the inertial forces are balanced by the Coulombic force. 


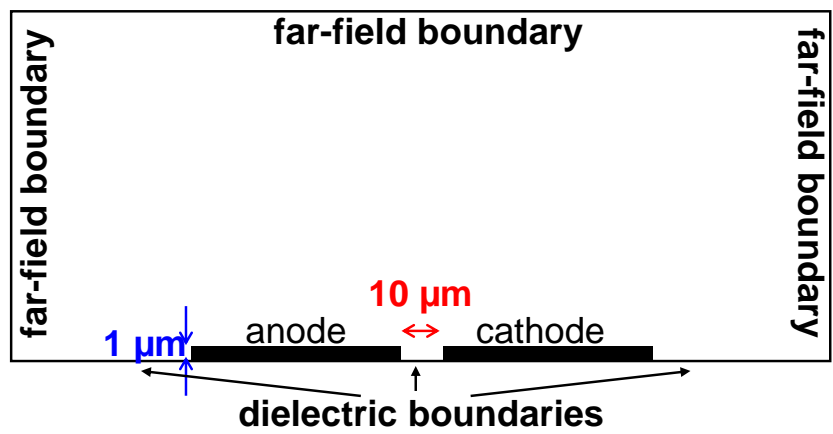

Figure 1 Schematic diagram of the basic simulation domain. 


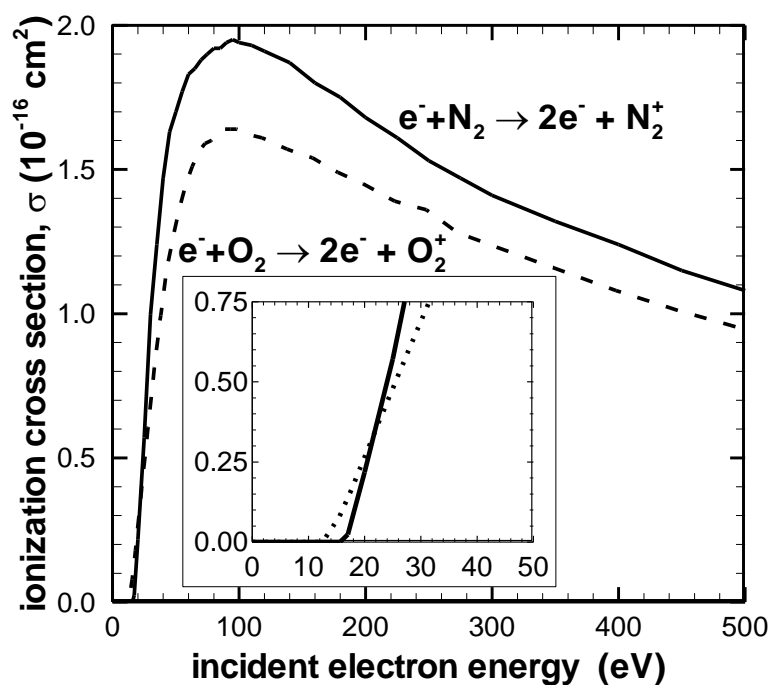

Figure 2 Collision cross sections as a function of electron energy for the ionization of $\mathrm{N}_{2}$ and $\mathrm{O}_{2}$. The inset shows a magnified view to clearly illustrate the minimum energy required for ionization. 


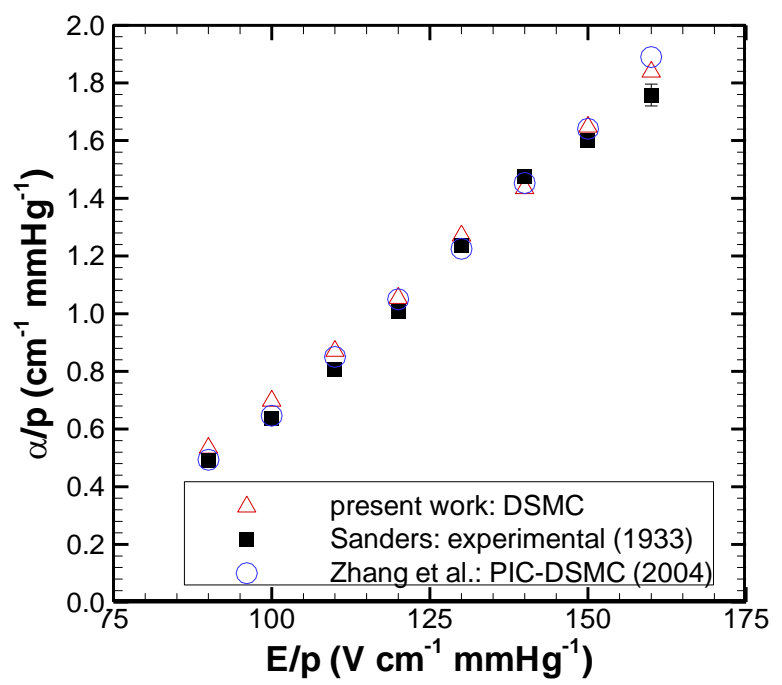

Figure 3 DSMC values of Townsend's first ionization coefficient as compared to the experimental values of Sanders [52] and the PIC-MCC values of Zhang et al. [5]. 


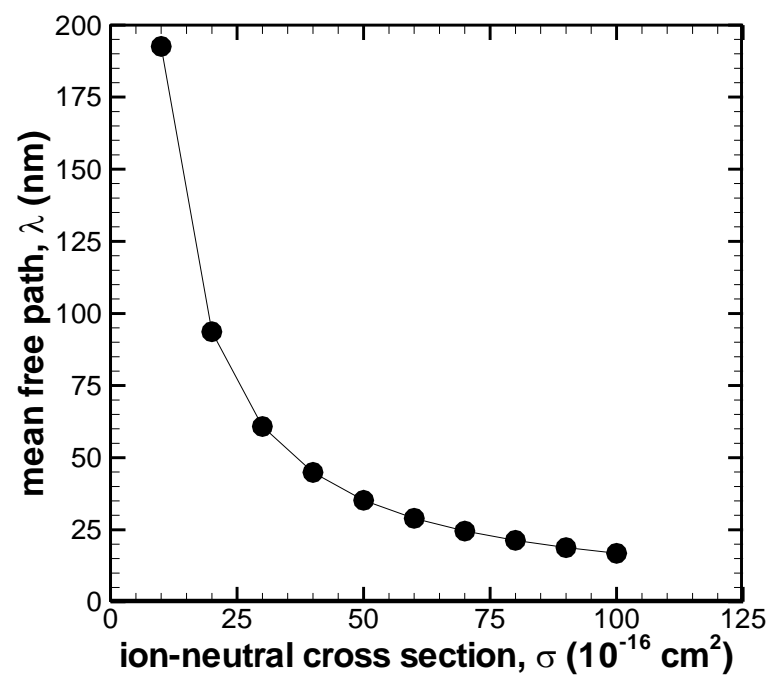

Figure 4 Variation of mean free path with collision cross section for ion-neutral collisions. 


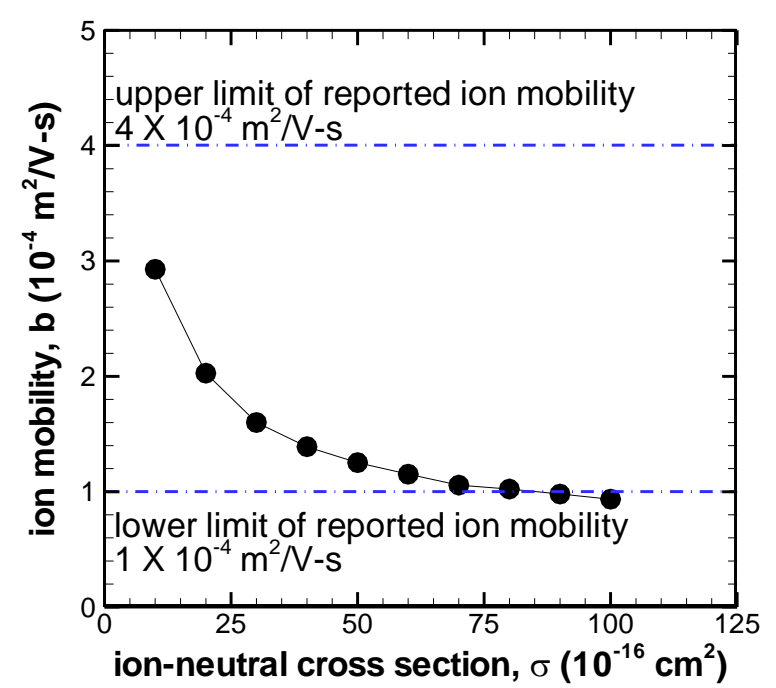

Figure 5 Variation of ion mobility with collision cross section for ion-neutral collisions along with values reported from the literature $[58,59]$. 


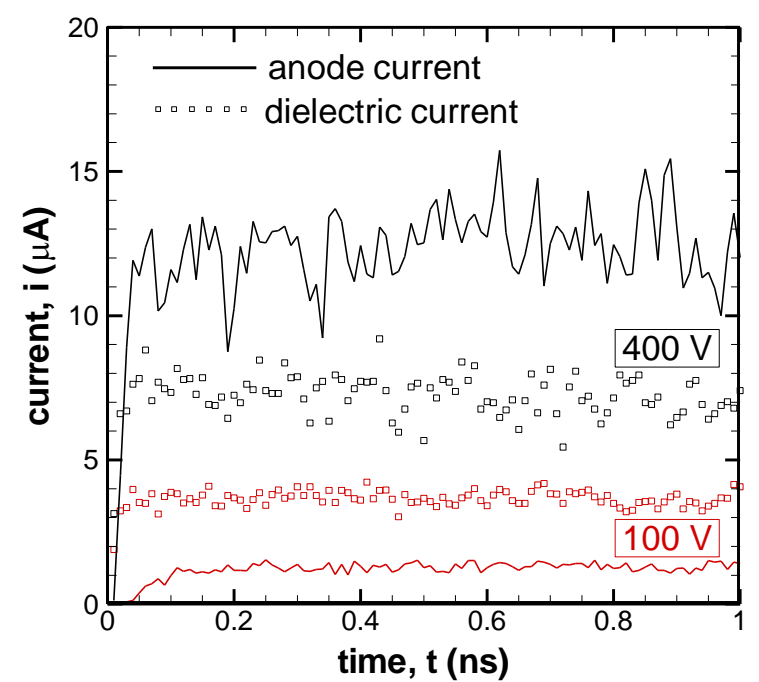

Figure 6 Electron current evolutions with time for an emission current of $5 \mu \mathrm{A}$. 


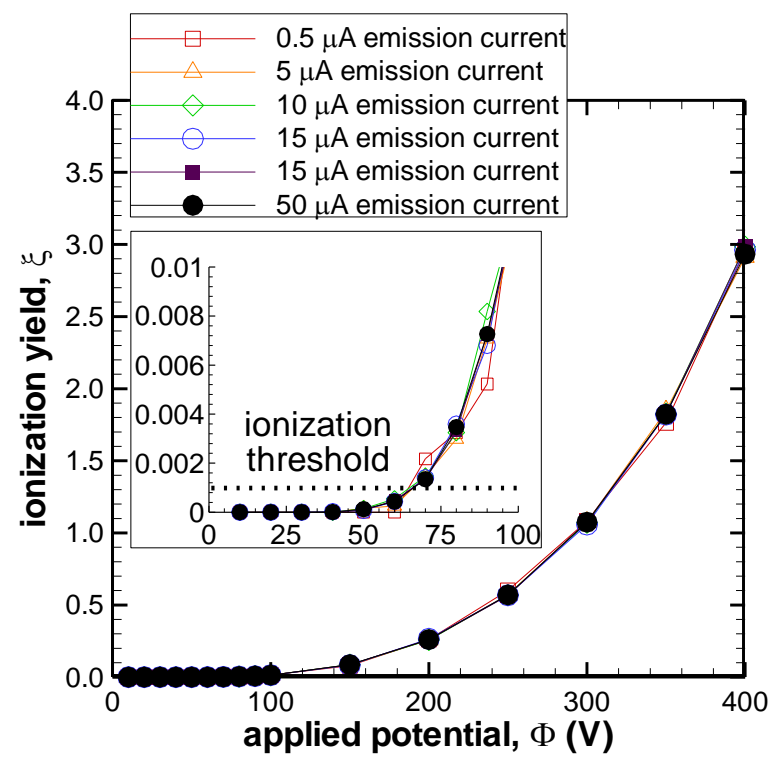

Figure 7 Ionization yield as a function of applied potential. 


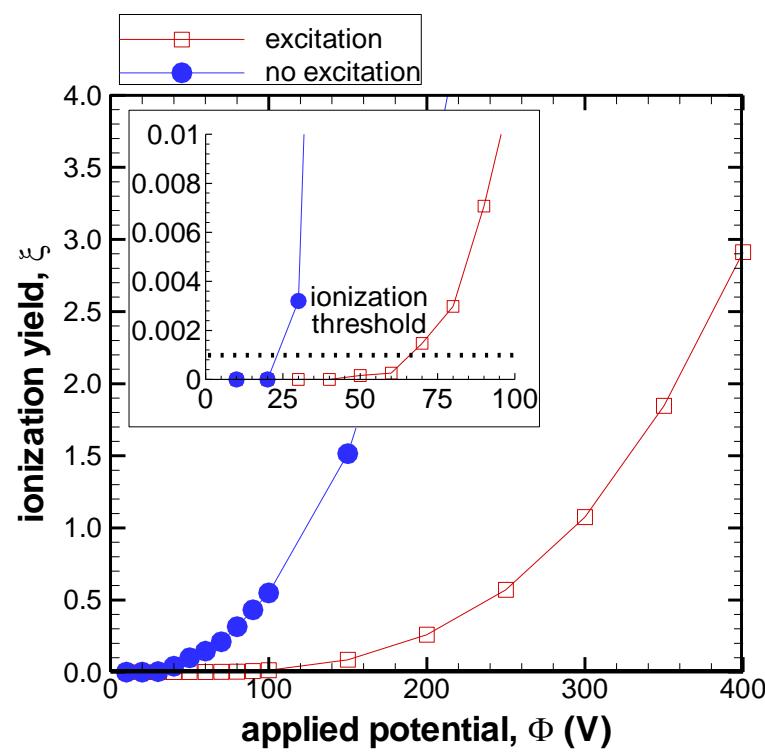

Figure 8 Ionization yield as a function of applied potential with the artificial condition of no energy loss during excitation reactions. 

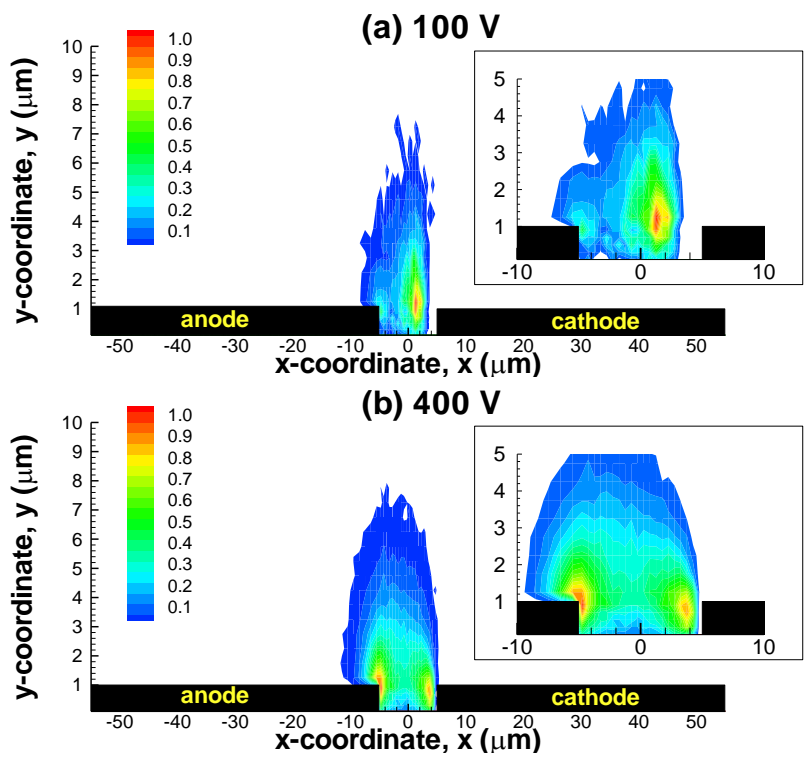

Figure 9 Ion generation concentration contours under applied potentials of (a) $100 \mathrm{~V}$, and (b) $400 \mathrm{~V}$, for an emission current of $5 \mu \mathrm{A}$. Each ion concentration plot, (a) and (b), is normalized to its respective maximum value. The insets are magnified views of the gap between the electrodes. 


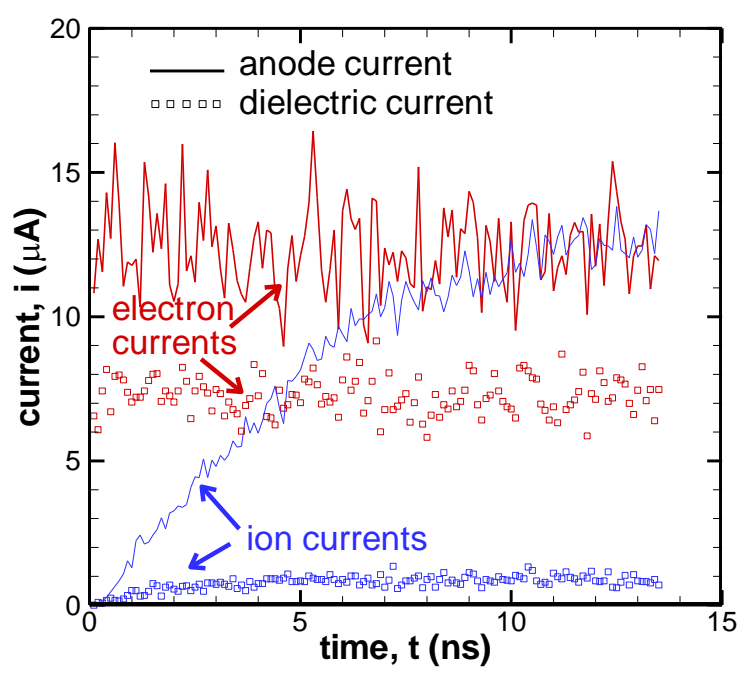

Figure 10 Ion and electron current evolution with time under a $400 \mathrm{~V}$ applied potential for an emission current of $5 \mu \mathrm{A}$. 


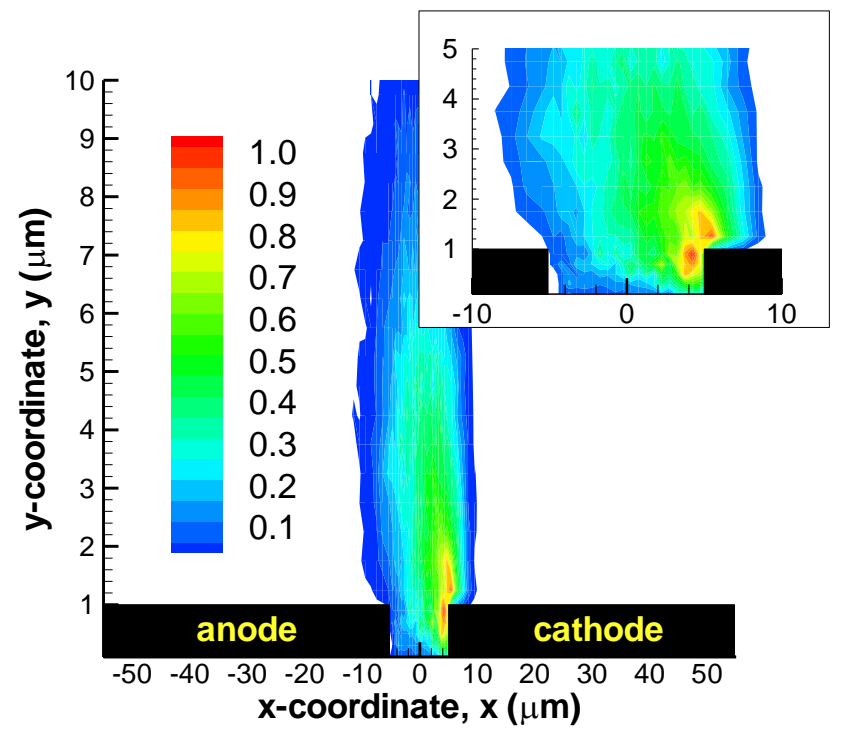

Figure 11 Normalized, steady ion concentration contours, $\rho_{\text {ion }} / \rho_{\text {ion,max }}$, under a $400 \mathrm{~V}$ applied potential for an emission current of $5 \mu \mathrm{A}$. The inset is a magnified view of the gap between the electrodes. 


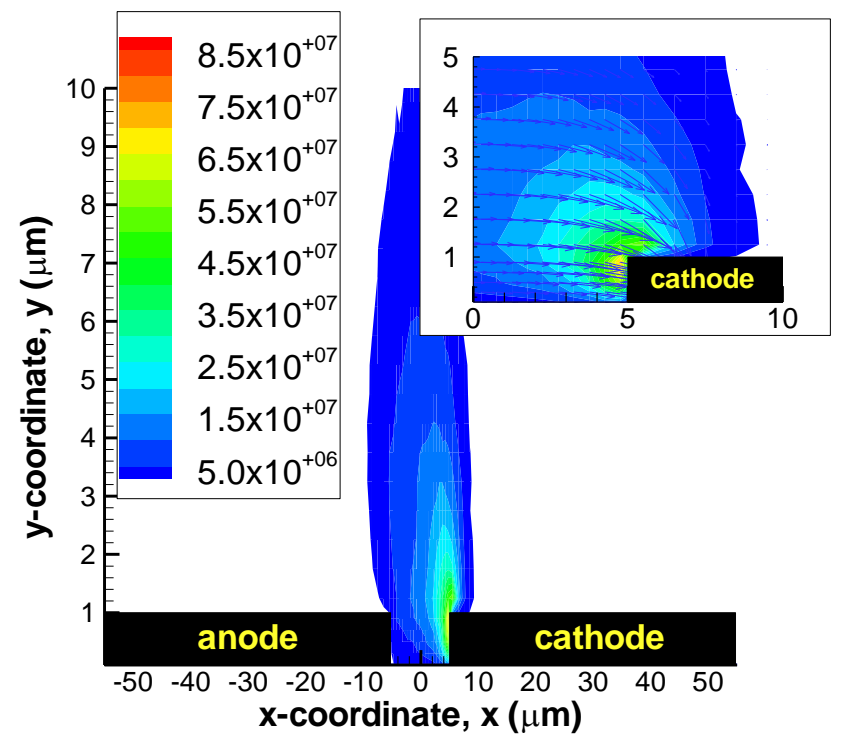

Figure 12 Body force magnitude contours (in $N / m^{3}$ ) under a $400 \mathrm{~V}$ applied potential with a $50 \mu \mathrm{A}$ emission current. The inset is a magnified view of the gap between the electrodes. 


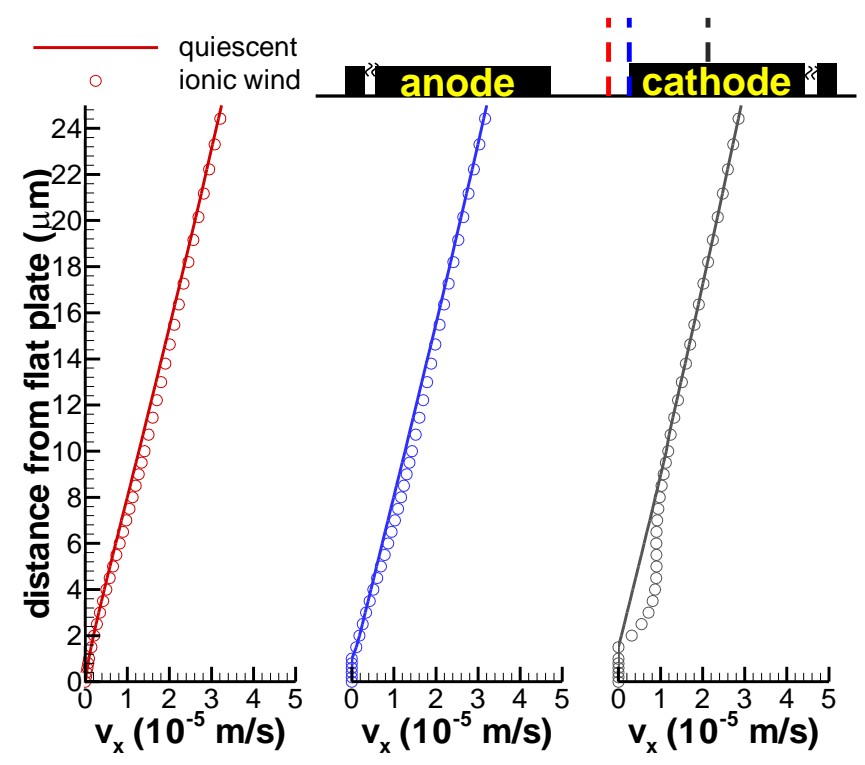

Figure 13 Boundary layer profiles for quiescent air as approximated by $10^{-4} \mathrm{~m} / \mathrm{s}$ flow and a $400 \mathrm{~V} / 50$ $\mu \mathrm{A}$ emission current ionic wind at three locations. The locations are denoted by the small graphic on the plot. 


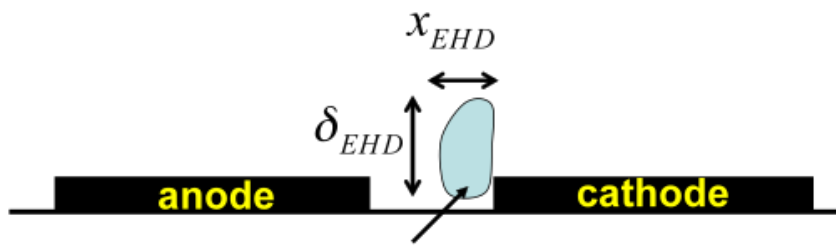

region over which

body force acts

Figure 14 Basic geometric definitions for the scale analysis. 


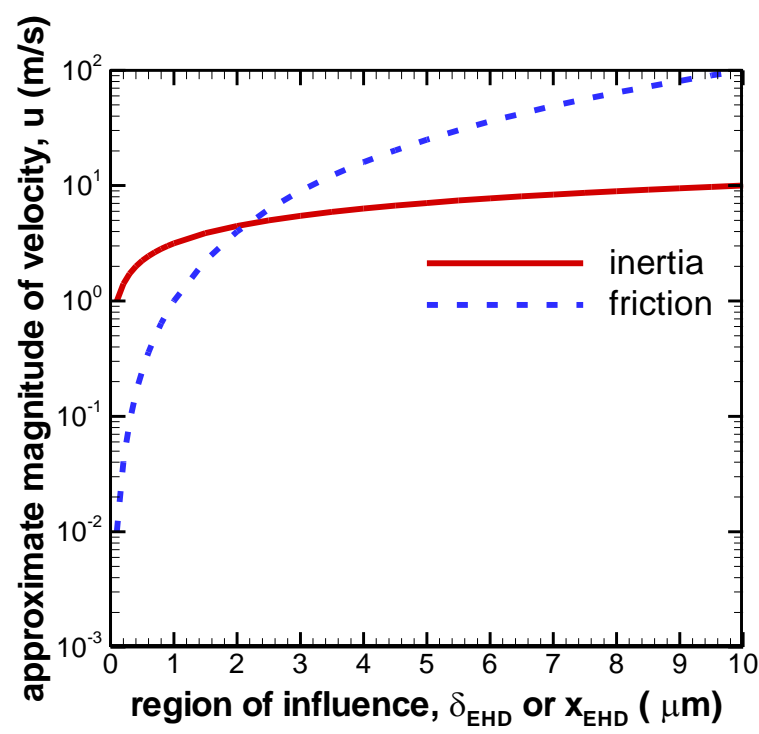

Figure 15 Approximate magnitude of induced velocity as a function of the region of influence. The dashed line refers to the induced velocity when the friction forces are balanced by the Coulombic force, and the solid line refers to the induced velocity when the inertial forces are balanced by the Coulombic force. 